Gut and Liver, Vol. 9, No. 5, September 2015, pp. 679-684

\title{
Evaluation of the Feasibility and Efficacy of Forward-Viewing Endoscopic Ultrasound
}

\author{
Seohyun Lee*, Dong Wan Seo*, Jun-Ho Choi ${ }^{\dagger}$, Do Hyun Park*, Sang Soo Lee*, Sung Koo Lee*, and Myung-Hwan Kim* \\ *Division of Gastroenterology, Department of Internal Medicine, Asan Medical Center, University of Ulsan College of Medicine, Seoul, and \\ ${ }^{\dagger}$ Division of Gastroenterology, Department of Internal Medicine, Dankook University College of Medicine, Cheonan, Korea
}

Background/Aims: We aimed to evaluate the feasibility and efficacy of a forward-viewing linear endoscopic ultrasound (FV-EUS) in diagnostic EUS procedures compared to standard oblique-viewing EUS (OV-EUS). Methods: This study was a prospective, randomized study that permitted crossover. Fifty-one patients with subepithelial pancreatobiliary and upper gastrointestinal lesions underwent FV-EUS and OV-EUS sequentially, in random order. The EUS visualization was performed by a novice endosonographer, and the image quality of specific lesions was scored by an expert endosonographer. If fine-needle aspiration (FNA) was indicated, it was performed using both echoendoscopes by an expert endosonographer. Results: Both of the EUS procedures had similar visualization times and image quality. In general, the visualization time was inversely related to the diameter of the specific lesions. In subepithelial lesions of the stomach and duodenum, the visualization time $(98.8 \pm 62.2$ seconds vs $139.0 \pm 66.6$ seconds, $p=0.008)$ and image quality $(4.1 \pm 1.3$ vs $3.3 \pm 1.7, p=0.02$ ) of FV-EUS were significantly superior to OV-EUS. FV-EUS-guided FNA of pancreatic masses was successful in seven patients (87.5\%). Conclusions: FV-EUS may increase the ease of access to gastrointestinal subepithelial lesions compared to conventional OV-EUS. The performance of FV-EUS for evaluating pancreatobiliary diseases and performing interventions was comparable to conventional OVEUS. (Gut Liver 2015;9:679-684)

Key Words: Endoscopic ultrasound guided intervention; Forward-viewing endoscopic ultrasound; Oblique-viewing endoscopic ultrasound

\section{INTRODUCTION}

The ability of echoendoscope technology to access extraluminal lesions under direct sonographic visualization increases both the technical success rate and safety profile of procedures. Traditionally, endoscopic ultrasound (EUS) and EUS-guided fine-needle aspiration (EUS-FNA) have been performed with an oblique-viewing (OV) EUS (OV-EUS). However, the maneuverability of the OV-EUS in the duodenum, particularly for sampling the pancreatic head and uncinate process lesions, can be challenging. ${ }^{1}$ A recently developed forward-viewing (FV) linear EUS (FV-EUS) features an ultrasonographic field of view almost coaxial to the exit path of the working channel, allowing the use of needles and other devices in a collinear fashion similar to a gastroscope.

Conventional EUS-FNA was introduced in the early 1990s and has since become the gold standard algorithm in evaluating suspicious masses adjacent to the gastrointestinal (GI) tract. ${ }^{2}$ Due to the surge in new applications for EUS-guided interventions, a FV-EUS has been developed.

The FV-EUS has an electrical curved linear array of $120^{\circ}$ forward endoscopic views. It has a 3.7-mm working channel without an elevator, and thus allows the exit of a FNA needle or any other accessories parallel to the longitudinal axis of the EUS. The EUS frequencies can be varied from 5 to $10 \mathrm{MHz}$, and the scanning plane direction is parallel to its insertion, with a $90^{\circ}$ scanning range. The FV-EUS was originally designed for therapeutic procedures. It has been reported to be useful for EUSFNA in specific circumstances due to its unique characteristics, ${ }^{1,3}$ but the utility of FV-EUS has only been reported anecdotally. Therefore, the aim of this study was to evaluate the feasibility and the efficacy of FV-EUS for evaluation of pancreaticobiliary and subepithelial lesions compared to conventional OV-EUS us-

\footnotetext{
Correspondence to: Dong Wan Seo

Division of Gastroenterology, Department of Internal Medicine, Asan Medical Center, University of Ulsan College of Medicine, 88 Olympic-ro 43gil, Songpa-gu, Seoul 138-736, Korea

Tel: +82-2-3010-3192, Fax: +82-2-3010-8043, E-mail: dwseoamc@amc.seoul.kr

Received on October 13, 2014. Revised on December 4, 2014. Accepted on December 4, 2014. Published online June 19, 2015 pISSN 1976-2283 eISSN 2005-1212 http://dx.doi.org/10.5009/gnl14394

@ This is an Open Access article distributed under the terms of the Creative Commons Attribution Non-Commercial License (http://creativecommons.org/licenses/by-nc/4.0) which permits unrestricted non-commercial use, distribution, and reproduction in any medium, provided the original work is properly cited.
} 
ing a prospective, randomized, and cross-over study design.

\section{MATERIALS AND METHODS}

\section{Patients}

Between December 2012 and March 2013, patients referred for evaluation of pancreaticobiliary diseases and GI subepithelial lesions were prospectively enrolled in the study (Fig. 1). The exclusion criteria were as follows: pregnancy, bleeding tendency (prothrombin time INR $>1.5$ or platelet $<80,000 / \mu \mathrm{L}$ ), and critically ill patients. In the study period, 62 patients were enrolled and 11 patients refused participation. Study approval was provided by the Ethics Committees of the Asan Medical Center, University of Ulsan College of Medicine. Written informed consent for participation was obtained from all enrolled patients.

\section{Observers}

Patients were placed in a left lateral decubitus position and all EUS procedures were performed under conscious sedation with intravenous midazolam and propofol according to the judgment of the endoscopist. Both the OV-EUS (GF-UE260-AL5, GF-UCT240; Olympus Medical System, Tokyo, Japan) and the newly developed FV-EUS (GIF-Y-0007-UCT; Olympus Medical System) were used. The sequence in which these techniques were employed was randomly assigned.

For the study protocol, two groups were formed depending on the experience of the examiner. The first examiner (expert) was a highly experienced endosonographer who had performed $>4,000$ EUS examinations related to pancreaticobiliary disease. The second examiner (novice) was a junior gastroenterology fellow who had performed <50 EUS examinations. Only the information regarding reason for referral was revealed to the examiners. The EUS was entirely performed by the fellow (novice examiner) without any hands-on assistance from the supervisors. Color Doppler EUS was used to assess involvement of vessels. The visualization time was defined as the time from intubation of the upper esophageal sphincter until visualization of the target lesion. If the time to visualize the lesions took more than 200 seconds, it was considered a failed procedure. The EUS image quality of specific lesions was scored using a five-point scoring system by an expert endosonographer, as follows: 1 , no visualization; 2, poor visualization; 3, fair visualization; 4, good visualization; 5, excellent visualization.

The EUS-FNA was performed by the expert endosonographer. Usefulness and feasibility of EUS-FNA were evaluated with each of the following four standards: visual field, targeting, needle puncture, and manipulation. A good visual field was defined by a high quality of image and successful striking of the target by the fine needle, whether or not the visualization of the lesion was well maintained. Precise targeting was defined as the needle passing and adjusting properly. A correct needle puncture was defined as the fine needle sticking accurately to the lesion without altered visualization throughout the procedure. A positive hands-on manipulation was defined as the needle and accessory kit operating easily after needle puncture. All four standards were rated as yes or no.

\section{Statistical analysis}

Continuous variables were reported as means with standard deviations or medians with interquartile ranges (IQR), while categorical variables were reported as proportions with respective percentages. Differences between categorical variables were tested using Pearson chi-square test or Fisher exact test, and continuous variables were tested using Student two-tailed test (normal distribution) or the Mann-Whitney U test (skewed distribution). To analyze the presence of random effects and

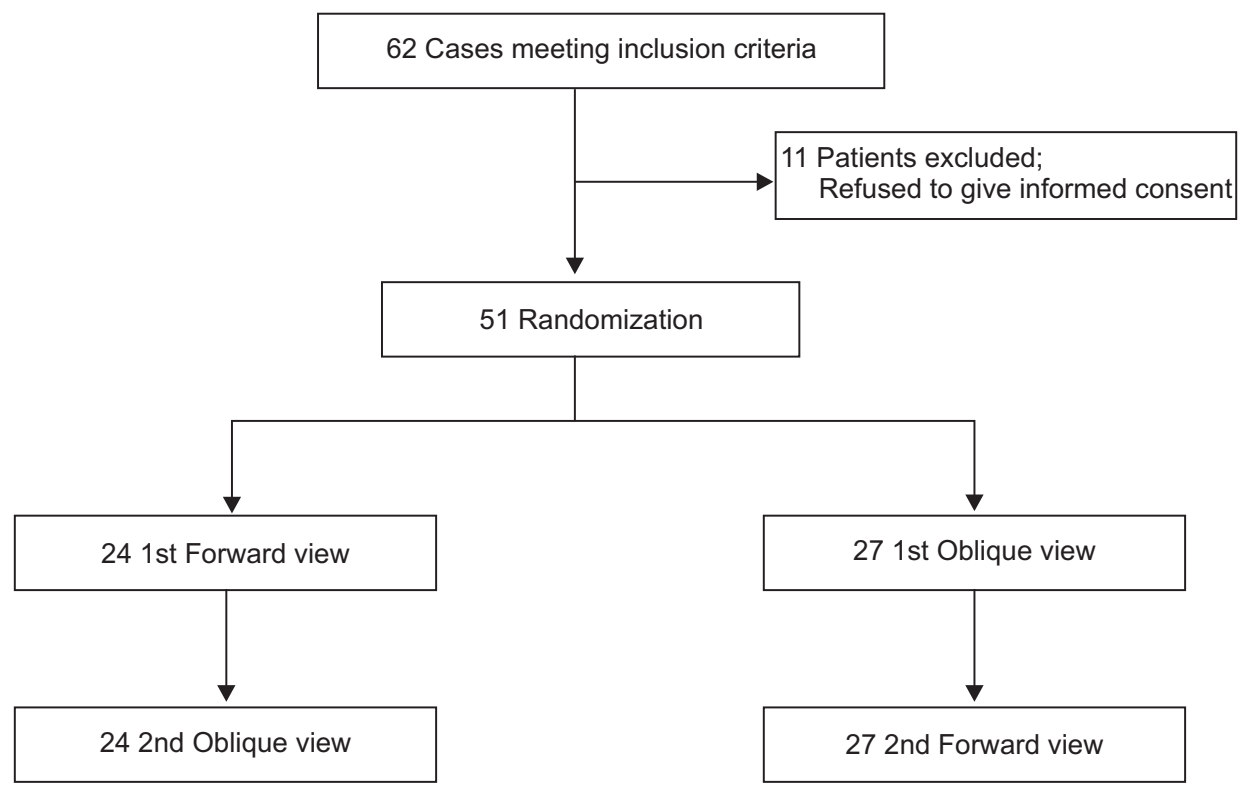

Fig. 1. Flow chart of the study design. 
to introduce correlations of repeated measures, a linear mixed model and cumulative logit analysis model with generalized estimating equation were tested. A p-value of $<0.05$ was regarded as statistically significant and data were analyzed with the SPSS software version 19.0 (SPSS Inc., Chicago, IL, USA).

\section{RESULTS}

\section{Visualization of EUS}

A total of 51 patients were analyzed (Fig. 1). The mean age was $60.4 \pm 11.5$ years; $49 \%$ were male. The examination was undertaken at the following sites: pancreatic head $(n=14)$, pancre-

Table 1. Patient and Lesion Characteristics

\begin{tabular}{lc}
\hline \multicolumn{1}{c}{ Characteristic } & Value \\
\hline No. of patients & 51 \\
Age, yr & $60.4 \pm 11.5$ \\
Sex, male/female & $25 / 26(49 / 51)$ \\
Largest diameter on EUS, mean \pm SD (IQR), mm & $20 \pm 14.1(10.3-26.8)$ \\
Organs/structures & \\
Pancreatic head & 14 \\
Pancreatic body/tail & 14 \\
Gallbladder & 6 \\
Stomach, duodenum subepithelial tumor & 16 \\
Common bile duct & 1 \\
\hline
\end{tabular}

Data are presented as mean \pm SD or number (\%) unless otherwise indicated. EUS, endoscopic ultrasound; IQR, interquartile range. atic body or tail $(n=14)$, gallbladder $(n=6)$, gastric subepithelial lesion $(n=6)$, duodenal subepithelial lesion $(n=10)$, and common bile duct $(n=1)$. The mean size of the lesions was $20 \pm 14.1 \mathrm{~mm}$ in largest dimension (Table 1).

The total mean visualization time of OV-EUS and FV-EUS was $119.8 \pm 56.5$ and $108.9 \pm 54.0$ seconds, respectively. If OVEUS or FV-EUS was performed first, the mean visualization times of OV-EUS and FV-EUS were 129.8 \pm 58.4 and 121.5 \pm 51.4 seconds, respectively. If OV-EUS or FV-EUS was performed second, the corresponding mean visualization times were 108.6 \pm 53.4 and $97.7 \pm 54.9$ seconds, respectively. When applying a linear mixed model, there was no time difference in visualization $(\mathrm{p}=0.144)$.

Specifically, the mean visualization times for pancreas head lesions of FV-EUS and OV-EUS were $115.5 \pm 44.7$ and $104.6 \pm 39.6$ seconds, respectively $(\mathrm{p}=0.310)$, and for pancreas body and tail lesions they were $99.6 \pm 51.8$ and $89.4 \pm 40.4 \mathrm{sec}-$ onds, respectively $(\mathrm{p}=0.245)$. The visualization times of FV-EUS and OV-EUS for subepithelial lesions of the stomach and duodenum were $98.8 \pm 62.2$ and $139.0 \pm 66.6$ seconds, respectively $(\mathrm{p}=0.008)$, and for gallbladder lesions were $150.8 \pm 53.5$ and $136.7 \pm 54.3$ seconds, respectively $(\mathrm{p}=0.104)$ (Table 2$)$.

Considering visualization, OV-EUS showed 58.1\% ( $n=25)$ of excellent visualization, $37.2 \%(\mathrm{n}=16)$ of good visualization, and 4.7\% $(n=2)$ with fair visualization. The FV-EUS showed 52.2\% $(n=24)$ of excellent visualization, 45.7\% $(n=21)$ of good visualization and $2.1 \%(\mathrm{n}=1)$ with fair visualization (Table 3$)$. The cumulative logic model with generalized estimating equation showed FV-EUS was not inferior to OV-EUS (odds ratio [OR],

Table 2. Visualization Time in FV-EUS and Conventional OV-EUS

\begin{tabular}{lccc}
\hline Organ/structure & FV-EUS & OV-EUS & p-value \\
\hline Pancreatic head & $115.5 \pm 44.7(75.0-162.5)$ & $104.6 \pm 39.6(52.5-145.5)$ & 0.310 \\
Pancreas body/tail & $99.6 \pm 51.8(57.0-142.5)$ & $89.4 \pm 40.4(55.5-127.5)$ & 0.245 \\
GI SET & $98.8 \pm 62.2(30.3-150)$ & $139.0 \pm 66.6(83.8-200)$ & 0.008 \\
Gallbladder & $150.8 \pm 53.5(98.75-200)$ & $136.7 \pm 54.3(100-170)$ & 0.104 \\
\hline
\end{tabular}

Data are presented as mean \pm SD (interquartile range).

FV-EUS, forward-view endoscopic ultrasound; OV-EUS, oblique-view endoscopic ultrasound; GI SET, gastrointestinal (stomach and duodenum) subepithelial tumor.

Table 3. Baseline Image-Quality Characteristics

\begin{tabular}{|c|c|c|c|c|c|}
\hline & Method & Nonvisualized & Fair & Good & Excellent \\
\hline \multirow[t]{2}{*}{ First exam } & OV-EUS & $5(18.5)$ & 0 & $10(37.0)$ & $12(44.5)$ \\
\hline & FV-EUS & $3(12.5)$ & $1(4.2)$ & 8 (33.3) & $12(50.0)$ \\
\hline \multirow[t]{2}{*}{ Second exam } & OV-EUS & $3(12.5)$ & $2(8.3)$ & $6(25.0)$ & $13(54.2)$ \\
\hline & FV-EUS & $2(7.4)$ & 0 & $13(48.1)$ & $12(44.5)$ \\
\hline \multirow[t]{2}{*}{ Total } & OV-EUS & $8(15.7)$ & $2(3.9)$ & $16(31.4)$ & 25 (49.0) \\
\hline & FV-EUS & $5(9.8)$ & $1(2.0)$ & 21 (41.2) & $24(47.0)$ \\
\hline
\end{tabular}

Data are presented as number (\%).

OV-EUS, oblique-view endoscopic ultrasound; FV-EUS, forward-view endoscopic ultrasound. 

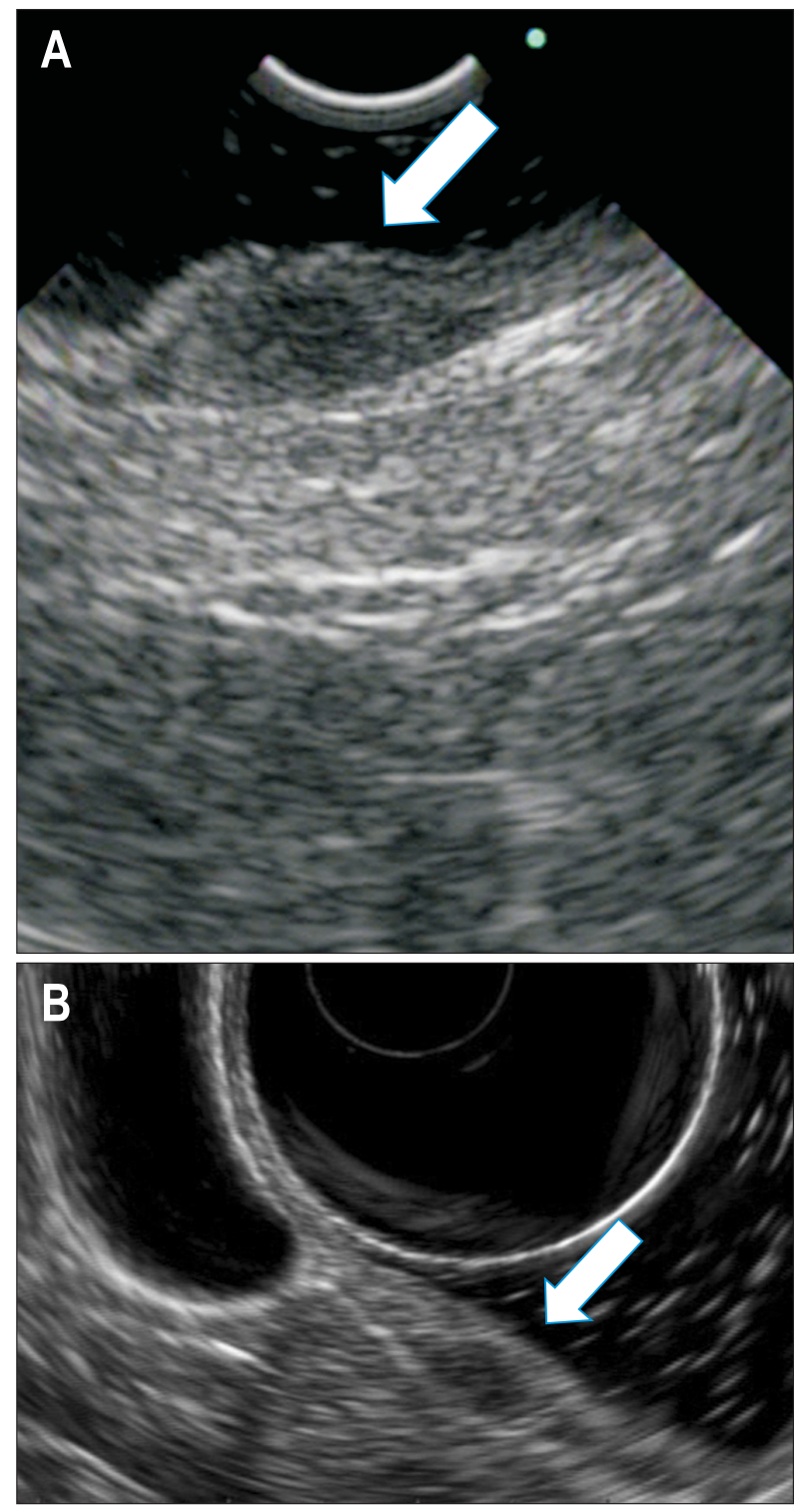

Fig. 2. Endoscopic ultrasound images of a duodenal subepithelial lesion with a forward-view endoscopic ultrasound (A) and an obliqueview endoscopic ultrasound (B). The forward-view endoscopic ultrasound yielded superior image quality.
0.83; 95\% confidence interval [CI], 0.43 to $1.62 ; \mathrm{p}=0.59$ ).

In detail, the mean scores of image quality for pancreas head lesions of FV-EUS and OV-EUS were $4.4 \pm 0.6$ and $4.4 \pm 0.6$, respectively $(\mathrm{p}=1.00)$, and for pancreas body and tail lesions were $4.6 \pm 0.5$ and $4.6 \pm 1.1$, respectively $(p=0.71)$. The image quality scores of FV-EUS and OV-EUS for subepithelial lesions of the stomach and duodenum were $4.1 \pm 1.3$ and $3.3 \pm 1.7$, respectively ( $\mathrm{p}=0.02$ ) (Fig. 2), and for gallbladder lesions were $3.3 \pm 1.9$ and $4.2 \pm 1.6$, respectively $(\mathrm{p}=0.1)$ (Table 4$)$.

One pancreatic body mass lesion was visualized only with FV-EUS, and one gallbladder lesion was visualized only with OV-EUS. The lesions were relatively small such that it was not easy for the novice endosonographer to trace the cystic duct of gallbladder. FV-EUS allows for better recognition of several features of chronic pancreatitis such as hyperechoic margins of the pancreatic duct, subtle lobularity of the parenchyma, small cystic changes in the parenchyma, and side branch duct ectasia than OV-EUS.

Among 16 cases of subepithelial lesion, two stomach subepithelial lesions and one duodenum subepithelial lesion failed to be visualized with the OV-EUS, whereas all subepithelial lesions were visualized with FV-EUS. One common bile duct mass lesion failed to be visualized by the novice endosonographer due to the difficulty in anatomical tracing (Table 5).

\section{EUS-FNA}

EUS-guided interventions were conducted in 12 patients

Table 4. Mean Imaging Quality Scores* with FV-EUS and Conventional OV-EUS

\begin{tabular}{lccc}
\hline \multicolumn{1}{c}{ Organ/structure } & FV-EUS & OV-EUS & p-value \\
\hline Pancreatic head & $4.4 \pm 0.6$ & $4.4 \pm 0.6$ & 1.00 \\
Pancreatic lesion & $4.6 \pm 0.5$ & $4.6 \pm 1.1$ & 0.71 \\
Subepithelial tumor & $4.1 \pm 1.3$ & $3.3 \pm 1.7$ & 0.02 \\
Gallbladder & $3.3 \pm 1.9$ & $4.2 \pm 1.6$ & 0.1 \\
\hline
\end{tabular}

Data are presented as mean \pm SD.

FV-EUS, forward-view endoscopic ultrasound; OV-EUS, oblique-view endoscopic ultrasound.

*1, no visualization; 2, poor visualization; 3, fair visualization; 4, good visualization; 5, excellent visualization.

Table 5. Visualization of Pancreaticobiliary Lesions with an Forward-View Echoendoscope and a Conventional Echoendoscope by a Novice Endosonographer

\begin{tabular}{lccccc}
\hline \multicolumn{1}{c}{ Location } & $\begin{array}{c}\text { Total no. of } \\
\text { patients }\end{array}$ & $\begin{array}{c}\text { Visualization on } \\
\text { both FV- and OV-EUS }\end{array}$ & $\begin{array}{c}\text { Visualization on } \\
\text { OV-EUS only }\end{array}$ & $\begin{array}{c}\text { Visualization on } \\
\text { FV-EUS only }\end{array}$ & $\begin{array}{c}\text { Invisualization } \\
\text { on both FV- and OV-EUS }\end{array}$ \\
\hline Pancreatic head & 14 & 14 & - & - & - \\
Pancreatic body and tail & 14 & 13 & - & - & 1 \\
Gallbladder & 6 & 4 & - & 3 & - \\
Subepithelial tumor & 16 & 13 & - & - & 1 \\
Common bile duct & 1 & - & & - \\
\hline
\end{tabular}

FV-EUS, forward-view endoscopic ultrasound; OV-EUS, oblique-view endoscopic ultrasound. 


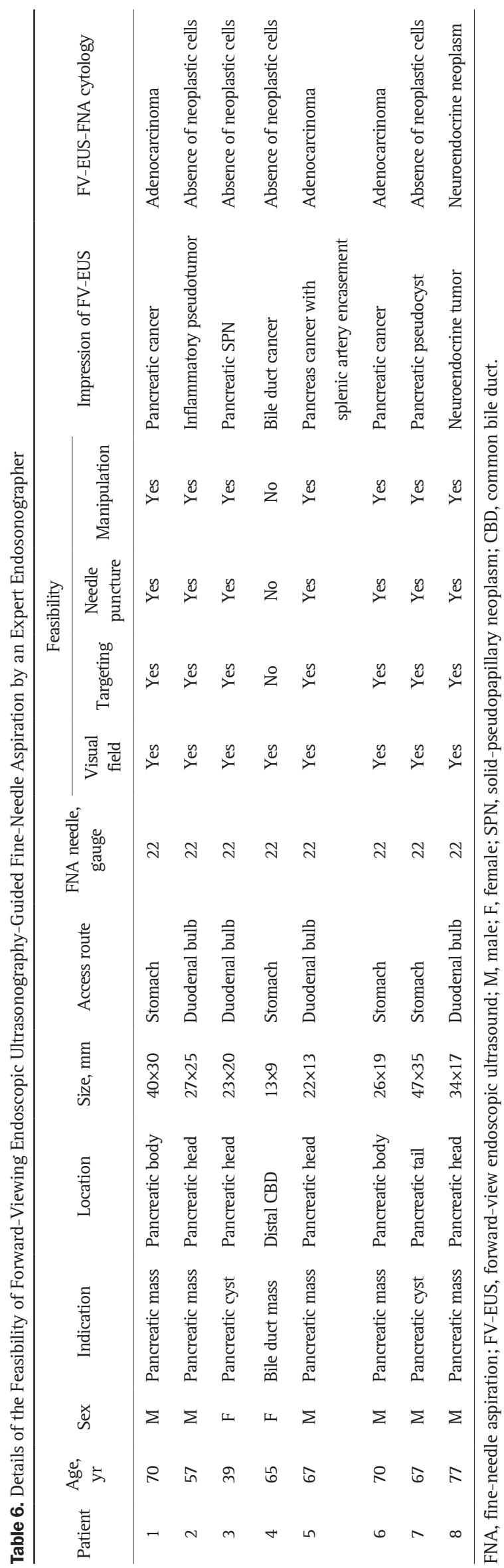

and mainly included FNA of a pancreatic mass by an expert endosonographer. EUS-FNA was performed with disposable 22-gauge needles (Echotip Ultra; Cook Medical Inc., WinstonSalem, NC, USA) inserted through the 3.7-mm working channel of the EUS. The clinical and procedural data of FV-EUS-guided FNA are summarized in Table 6. Visualization of the primary lesion by FV-EUS was obtained in all of the eight cases, and FV-EUS-guided FNA was performed successfully in seven patients (87.5\%). There were no procedure-related adverse events. Procedural indications included pancreatic mass detected on computed tomography (CT) or transabdominal ultrasonography $(n=5)$, pancreatic cyst seen on CT $(n=2)$, and intrabile duct mass noted on CT $(n=1)$. The lesions were located in the pancreatic head in four patients, in the pancreatic body in two patients, in the pancreatic tail in one patient and in the distal common bile duct in one patient. EUS-FNA using both FV- and OV-EUS failed to obtain diagnostic samples in the patient with a distal bile duct mass due to surgically altered anatomy and major vessel interposition. For this patient, percutaneous transhepatic biliary drainage was inserted and a biopsy was taken using choledochoscopy.

\section{DISCUSSION}

In this report, we have presented our initial experience in the evaluation of pancreatobiliary and subepithelial lesions with an FV-EUS, which has been developed with the aim of potentially expanding the therapeutic applications of EUS and EUS-guided interventions.

The access to the target lesion was faster in the FV-EUS than in the OV group, especially in subepithelial lesions. Overall, there was no significant difference in image quality between the two groups, but in subepithelial lesions, the FV-EUS cohort showed superior image quality. Once in contact with the intestinal wall, the smaller transducer of the FV-EUS, compared to that of the OV-EUS, allows scanning in a perpendicular direction relative to the insertion direction, which could be advantageous for clinical evaluation. ${ }^{4}$ Lesions in the pancreatic body and tail appeared easier to locate with a FV-EUS, whereas for pancreatic head and cystic duct lesions of diameter $<20 \mathrm{~mm}$, the image quality of FV-EUS was inferior to OV-EUS.

A major drawback of conventional OV-EUS is that endoscopic accessories must access the targeted lesions at an acute angle when they are deployed through the biopsy channel. This exerts force on the needle, which can often unintentionally result in the EUS being pushed away from the intestinal wall, leading to technical failure. Moreover, the tip of the EUS must always be maintained straight to permit an easy path for accessories, and this can be technically difficult to achieve, particularly when the EUS is in an acutely angulated position, such as in the gastric cardia, the duodenum, or the rectosigmoid junction. ${ }^{6}$ Another major disadvantage of the OV-EUS is that it requires reorienta- 
tion when switching from a sonographic to an endoscopic view, because the intestinal wall frequently opposes the EUS during interventions under sonographic guidance. ${ }^{7}$

On the contrary, FV-EUS enables a field of examination that is almost parallel to the endoscopic view, which makes it easier to deploy the needle and allows a greater penetrating force with a precise orientation of the needle. ${ }^{5}$ The needle could also be advanced through the working channel in the retroflexed position and handled easily by simply applying up and down deflection. In addition, FV-EUS has improved in upward function, which could be bent up to $180^{\circ}$, compared to $130^{\circ}$ for OV-EUS. This allows direct targeting of lesions in an easier position with the frontal exit of the needle. ${ }^{4}$

The FV-EUS scope maneuverability and stability, and the ease of performing FNA, were satisfactory and comparable to that of previous studies evaluating the yield of EUS-FNA. The limitations of the FV-EUS are that is has a narrower field of view than the OV-EUS, ${ }^{5}$ it does not have an elevator or a balloon, and the outer diameter of the distal end and the insertion tube are smaller. However, from our experience, it is possible to satisfactorily visualize and obtain high diagnostic yields. The forward-array image and the availability of Doppler imaging also allowed safer FNA procedures. We also found there were no limitations in targeting with FV-EUS.

In summary, our experience with FV-EUS for evaluation of pancreatobiliary and GI subepithelial diseases seems to be comparable to conventional OV-EUS. The narrow image does not preclude basic diagnostic maneuvers. Moreover, visualization was obtained faster with FV-EUS and it significantly increased the ease of access to GI subepithelial tumors. These FV-EUS features allowed facile procedures to be performed by a novice endosonographer. FV-EUS-guided FNA also showed no limitations in the hands of an expert endosonographer. This suggests that FV-EUS and FV-EUS-guided FNA could be useful adjuncts to conventional OV-EUS and OV-EUS-FNA.

\section{CONFLICTS OF INTEREST}

No potential conflict of interest relevant to this article was reported.

\section{ACKNOWLEDGEMENTS}

We would like to thank Olympus Medical System for the loan of the FV-EUS system.

\section{REFERENCES}

1. Eloubeidi MA. Initial evaluation of the forward-viewing echoendoscope prototype for performing fine-needle aspiration, Trucut biopsy, and celiac plexus neurolysis. J Gastroenterol Hepatol 2011;26:63-67.

2. Erickson RA. EUS-guided FNA. Gastrointest Endosc 2004;60:267279

3. Sepe PS, Moparty B, Pitman MB, Saltzman JR, Brugge WR. EUSguided FNA for the diagnosis of GI stromal cell tumors: sensitivity and cytologic yield. Gastrointest Endosc 2009;70:254-261.

4. Larghi A, Lecca PG, Ardito F, et al. Evaluation of hilar biliary strictures by using a newly developed forward-viewing therapeutic echoendoscope: preliminary results of an ongoing experience. Gastrointest Endosc 2009;69:356-360.

5. Fusaroli P, Cortecchia S, Caletti G. EUS-FNA using a forward-view echoendoscope in difficult cases. J Gastrointestin Liver Dis 2011; 20:216-217

6. Nguyen-Tang T, Shah JN, Sanchez-Yague A, Binmoeller KF. Use of the front-view forward-array echoendoscope to evaluate right colonic subepithelial lesions. Gastrointest Endosc 2010;72:606610.

7. Trevino JM, Varadarajulu S. Initial experience with the prototype forward-viewing echoendoscope for therapeutic interventions other than pancreatic pseudocyst drainage (with videos). Gastrointest Endosc 2009;69:361-365. 